Journal of Engineering and Applied Sciences 14 (Special Issue 3): 5962-5967, 2019

ISSN: 1816-949X

(C) Medwell Journals, 2019

\title{
Denoising of Image using Bilateral Filtering in Multiresolution
}

\author{
${ }^{1}$ Alaa Abid Muslam Abid Ali, ${ }^{1}$ Mohammed Iqbal Dohan and ${ }^{2}$ Saif Khalid Musluh \\ ${ }^{1}$ Departement of Multimedia, \\ ${ }^{2}$ Departement of Information System, University of Al-Qadisiyah, Al-Diwaniyah, Iraq \\ Alaa.abidmuslam@qu.edu.iq
}

\begin{abstract}
One of the very efficient and resource conservative image processing methodology is with the help of bilateral filters. This technique filters the image without the help of edge smoothing but it does employs spatial averaging in a non-linear way. The filtering technique discussed above is very much dependent on the parameters of its filters. A very slight change in filter parameter values effects the outputs and results in a most drastic manner. In this study, the researcher has worked on two contributions. In the applications concerning image denoising, the researcher has contributed in study of the parameter selection of bilateral filters which are optimal in nature. The contribution number two is about extending the present research, i.e., extension of the filters which are bilateral in nature. In this process, the bilateral filtering of images is applied to the lower frequency sub-bands which is also known as approximation sub-band. This sub-band is obtained by using the wavelet transformations. Hence, a new framework for image denoising will be created which will be combination of multiresolution bilateral filtering and wavelets transformation techniques. As a matter of fact, this combination is efficient in contradicting noise from an image.
\end{abstract}

$\underline{\text { Key words: Biletaral filters, wavelet transform, image denoising, employs, non-liner, multiresolution }}$

\section{INTRODUCTION}

Digital data, more preciously digital images are prone to noise from, so, many different sources. Display of characteristics which are non-uniformly spatial in nature are shown by some of the noise components such as Dark Signal Non-Uniformity aka (DSNU) or Photo-Response Non-Uniformity (PRNU). Because of the reason that the spatial pattern of the above mentioned noise does not vary with time, the noise is sometimes referred to as Fixed Pattern Noise (FPN). This contrasts with the temporal noise where the noise does not happen to have a fixed pattern. Some of the examples of temporal noise are reset noise, read noise, photon shot noise and dark current. Well there are many other characteristic as well which determines the noise, these factors includes type of sensors in the image capture device, dimensions of the pixel, temperature, time of exposure and speed of ISO. In the most general form, the digital noise varies with space and dependent on the channel. As we know that, the blue filters has got the lowest transmittance, blue colored channel is generally the nosiest of all. One of the very much neglected characteristic of digital noise in images is its spatial frequency. If we see Fig. 1, we can actually see the high-frequency aka fine-grain and low-frequency aka coarse grain fluctuations. It is comparatively easier to remove the noise which is of high frequency. Whereas it (a)

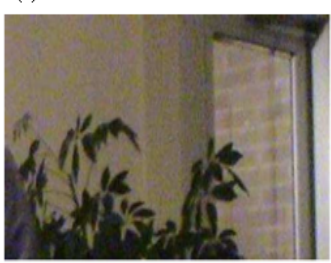

(c)

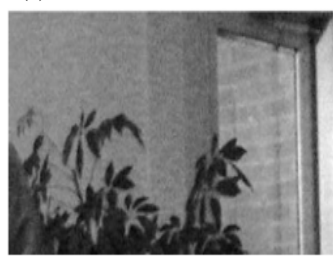

(b)

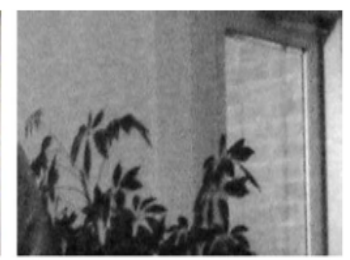

(d)

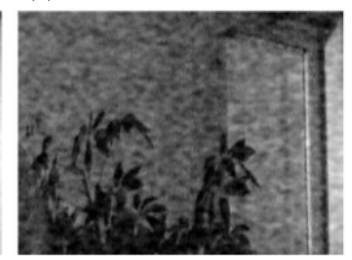

Fig. 1: a-d) Image and its red, green and blue channels

becomes very difficult to get hold of difference between the original message/image signal and a low frequency noise.

Over the course of time, there have been multiple image denoising methods developed and researched upon. The most popular methods of all is undoubtedly the use of wavelet transformation and thresholding. As per the official definition of wavelet thresholding, the signal under processing is decomposed into two components. The first one being approximation coefficients of the data

Corresponding Author: Alaa Abid Muslam Abid Ali, Department of Information System, College of Computer Science and IT, University of Al-Qadisiyah, Al-Diwaniyah, Iraq, Alaa.abidmuslam@qu.edu.iq 
which is also known as low-frequency components. The second one is its detailing coefficients which is also known as high-frequency components. Now, the most part of image information is stored in the large coefficients which is easier to denoise. Problematic area is denoising the detail sub-bands. This denoising is done by wavelet thresholding operation. Hence, wavelet thresholding operation is the key element of image denoising via. wavelets. There are a number of threshold strategies being researched, popular ones being VisuShrink (Donoho and Johnstone, 1994), SureShrink (Donoho et al., 1995) and BayesShrink (Chang et al., 2000). In the first one which is VisuShrink approach, the threshold is calculated which act universally on the noisy data. This threshold is actually a function of noise variance, however, the number of samples is created using the error measure of minimax approach. In the second one which is SureShrink thresholding approach, it works in relation with the risk estimator as proposed by Stein. In the final one which is Bayesian thresholding, the value of the threshold is determined in the Bayesian framework. These were some of the commonly used thresholding methods in wavelet thresholding. However, with the course of time the above mentioned methodologies have been improved by various methods, common ones being the correlation of wavelet coefficients at the interscale and intrascale level (Portilla et al., 2003; Pizurica and Philips, 2006; Sendur and Selesnick, 2002a, b; Luisier et al., 2007).

One of the most recent methods which is also quite popular now is bilateral filter (Lee, 1983). The basic methodology behind working of this filter is that is focuses on a local neighborhood of pixels and takes its weighted sum. The calculation of weights depends upon the intensity distance and spatial distance. This is a very good method as it preserves all the edges of the image data but on the other hand noise present in that local neighborhood gets leveled/averaged out.

There have been a lot of recent studies/journals which have actually connected the technique of multiresolution bilateral filtering with a lot another well established and tested techniques. By Elad (2002), it is very well mentioned that with the help of a extremely specific cost function the initial iteration of the algorithm of Jacobi is exactly similar to the bilateral filtering. Study like (Barash, 2002; Buades et al., 2006) states that bilateral filters and anisotropic diffusion are similar in nature (Lyu and Simoncelli, 2007; Elad and Aharon, 2006a, b; Mairal et al., 2008; Dabov et al., 2006; Dabov et al., 2007; Hirakawa and Parks, 2006).

So far, we have talked about the applications of bilateral filtering on only image denoising domain but that is not the only domain it works with. There are many other applications where bilateral filtering is being used. Retinex (Elad, 2005), image enhancements (Fattal et al., 2007), volumetric denoising (Wong et al., 2004) etc. are some of them.

\section{MATERIALS AND METHODS}

Bilateral filter's parameter seletion: It is evident from Donoho and Johnstone (1994) that the two essentials parameters to be used in bilateral filtering are intensity domain and spatial domain. The topic of selecting appropriate value for these parameters have not been answered properly while talking in theory. The study of Buades et al. (2006) finds out the first derivative of the signal and then checks out its behavior as compared to intensity domain by spatial domain values. Another method was discussed by Kervrann and Boulanger (2006) where the researcher hypothesis for a non-local means algorithm a method which is adaptive in nature along with neighborhood size selection. The size of the neighborhood is chosen such that the upper bound of local $\mathrm{L} 2$ risk is coming out to be bare minimum. But the thing to be noted here is that effect of intensity domain is not taken into consideration. This section of the study is to deal with the presentation of the study (which would be empirical in nature) of the parameter values and setting them up to an optimal range. These parameter values will be function of the noise variance. We might also see that the first parameter i.e., intensity domain parameter is much more important at criticality then spatial domain parameter (Yaroslavsky, 1985; Smith and Brady, 1997; Sochen et al., 2001).

In this study, we will demonstrate the experiment which was done to understand the relationship between domain parameter, spatial parameter and the noise standard deviation. We started with some test images and added zero mean white Gaussian noise to them. Then we apply our bilateral filter on this noisy image in which we just added the noise. This bilateral filter was applied on varied values of domain parameter and spatial parameter. Also, this very experiment was done multiple times, again with varied values of noise variances and based on that multiple MSEs (Mean Squared Value) were observed and noted. The experiment shows that the optimal value of spatial domain parameter does not change very much with the level of noise variance. However, when we compare the optimal value of intensity domain parameter, it does changes more than the previous parameter. On further calculations in the experiment, it was seen that a better range for spatial domain parameter is approximately 1.5-2.1. But the optimal value of intensity domain parameter changes drastically when the standard deviation of noise changes. The result is very much expected because the numerical value of intensity domain parameter is much smaller than the spatial domain 


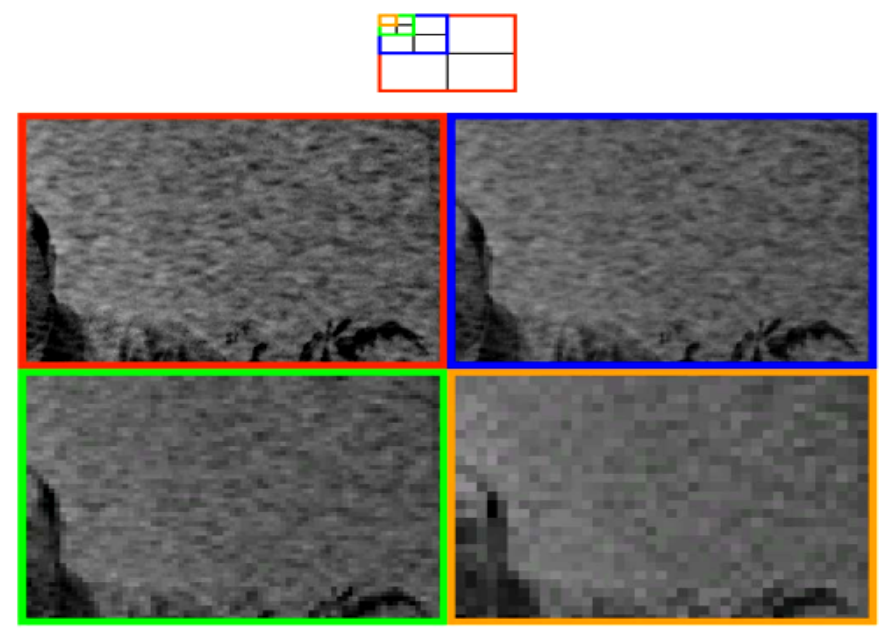

Fig. 2: Multiresolution properties of a coarse grain noise

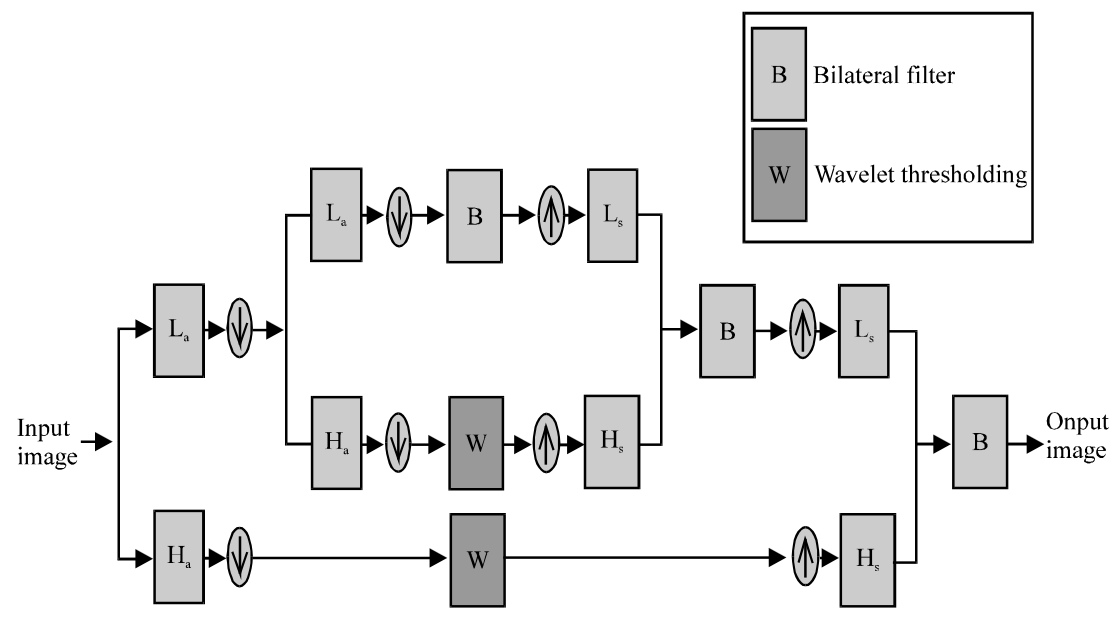

Fig. 3: Proposed methodology

parameter. Because of which the noisy data remains untouched, just like in the case of salt and pepper noise problem as seen in bilateral filter (Tomasi and Manduchi, 1998). When intensity domain parameter is large enough, spatial domain parameter becomes important. We should note that the value of spatial domain parameter should not be increased too much, it might result in over-smoothing of the noisy area and this will lead to decrease in MSE value (Sochen et al., 2001; Spira et al., 2007; Durand and Dorsey, 2002; Eisemann and Durand, 2004; Bennett and Mcmillan, 2005).

Framework for multiresolution denoiding of noisy image: As it is already discussed above in first section, only white noise does not exist in images, it may happen that the noise have many different spatial frequencies (fine grain and coarse grain). In various research studys, analyzing noisy images via. multiresolution analysis has proved to $\mathrm{b}$ e an important and efficient tool for removing noise. It makes it easier to separate image with noisy pixels. The image shown in Fig. 2 is evident enough to use the bilateral filtering technique in multiresolution framework. In the image, we can see the approximate sub-bands of a noisy image. The image also shows the transformation of coarse grain noise into fine grain noise when the image is decomposed into its sub-bands. This also shows that we can get rid of the coarse grain noise at lower level of sub-band (Paris and Durand, 2006; Acton, 1998).

The proposed framework as suggested by the researcher is shown in Fig. 3. We make use of wavelets decomposition method using which a signal is then decomposed into its frequency sub-bands. Before reconstructing the signal back, we apply bilateral filtering into the approximation sub-band. This advanced filter is now capable of removing low-frequency noise 
components, very different than the standard single-level bilateral filter (Tomasi and Manduchi, 1998). To sum the whole process, bilateral filters works in the approximation sub-band area and in this area some noise components can be identified and removed in an effective manner. Hence the new denoising concept combines two very efficient methods namely wavelet decomposition and bilateral filtering technique.

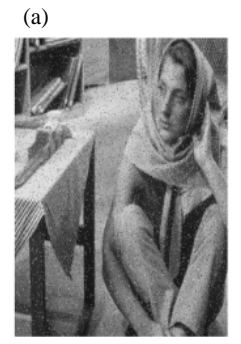

(c)
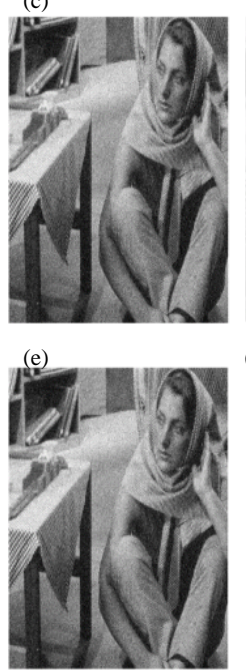

(g)

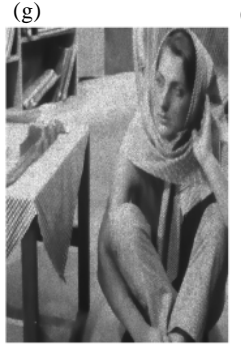

(b)

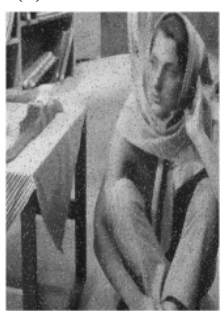

(d)
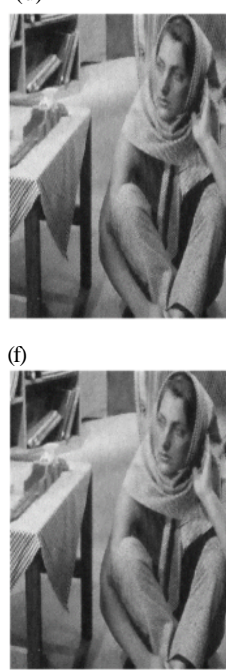

(i)

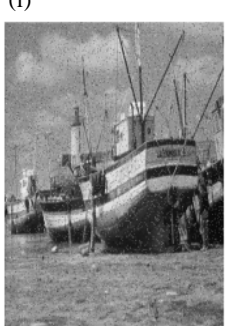

(k)

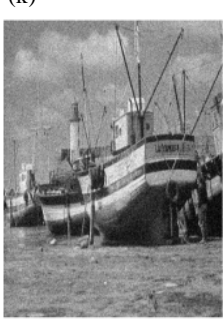

(m)

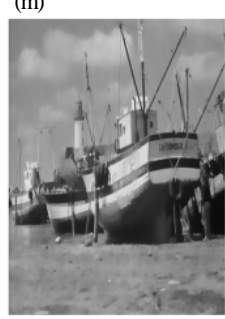

(o)

(h)

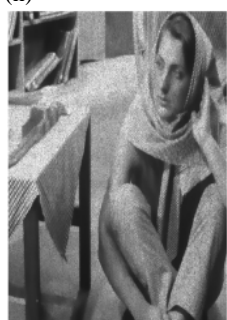

\section{RESULTS AND DISCUSSION}

Experiments were performed on the proposed scenario to check the efficiency and effectiveness of the scheme. To get the desired output, we picked few images and added noise to them. Then these images were denoised using the proposed scheme. Following are all the noisy images and their denoised counterparts (Fig. 4).

(j)

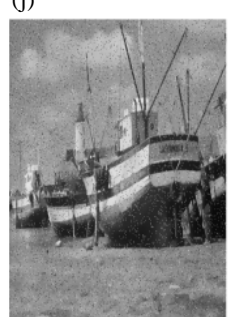

(1)

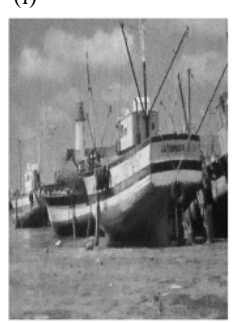

(n)
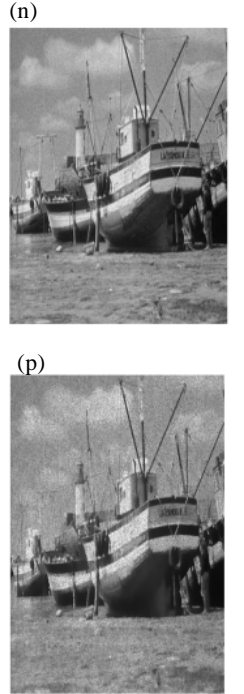

(q)

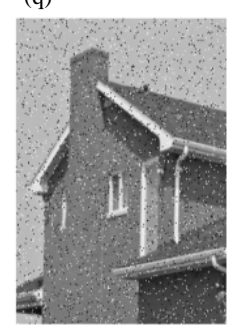

(s)

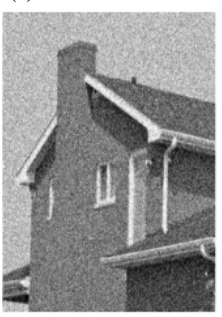

(u)

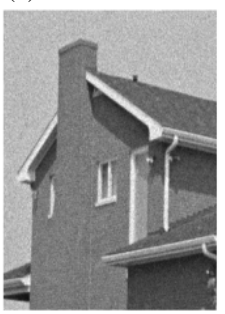

(w)

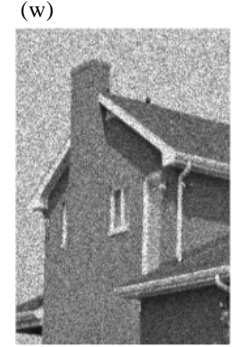

(r)

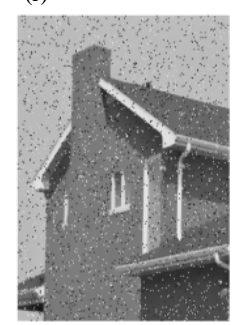

(t)

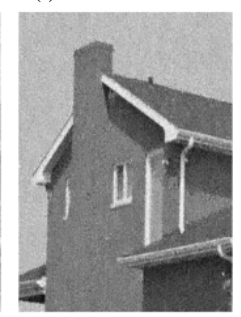

(v)

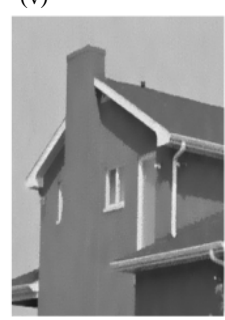

(x)

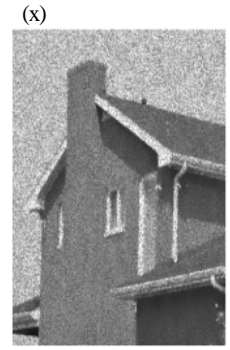

Fig. 4: The noisy image and denoised image: a) Noisy image: salt and pepper noise; b) Denoised image: salt and pepper noise; c) Noisy image: gaussian noise; d) Denoised image: gaussian noise; e) Noisy image: poisson noise; f) Denoised image: poisson noise; g) Noisy image: speckle noise; h) Denoised image: speckle noise; i) Noisy image: salt and pepper noise; j) Denoised image: salt and pepper noise; k) Noisy image: gaussian noise; 1) Denoised image: gaussian noise; m) Noisy image: poisson noise; n) Denoised image: poisson noise; o) Noisy image: speckle noise; p) Denoised image: speckle noise; q) Noisy image: salt and pepper noise; r) Denoised image: salt and pepper noise; s) Noisy image: gaussian noise; t) Denoised image: gaussian noise; u) Noisy image: poisson noise; v) Denoised image: poisson noise; w) Noisy image: speckle noise and x) Denoised image: speckle noise 


\section{CONCLUSION}

In this study, the main focus of the computation was on optimal value of the parameters that are to be used for bilateral filtering with image denoising being the main application. This would present a framework with multiresolution image denoising, not to forget that this scheme is an amalgam of wavelet processing and bilateral filtering.

\section{REFERENCES}

Acton, S.T., 1998. Multigrid anisotropic diffusion. IEEE. Trans. Image Process., 7: 280-291.

Barash, D., 2002. Fundamental relationship between bilateral filtering, adaptive smoothing and the nonlinear diffusion equation. IEEE. Trans. Pattern Anal. Mach. Intell., 24: 844-847.

Bennett, E.P. and L. Mcmillan, 2005. Video enhancement using per-pixel virtual exposures. Proceedings of the International Conference Special Interest Group on Computer Graphics and Interactive Techniques, July 31-August 4, 2005, Los Angeles, CA, USA pp: 845-852.

Buades, A., B. Coll and J.M. Morel, 2006. Neighborhood filters and PDE's. Numerische Math., 105: 1-34.

Chang, S.G., B. Yu and M. Vetterli, 2000. Adaptive wavelet thresholding for image denoising and compression. IEEE Trans. Image Process, 9: 1532-1546.

Dabov, K., A. Foi, V. Katkovnik and K. Egiazarian, 2006. Image denoising with block-matching and $3 \mathrm{D}$ filtering. Proceedings of the 2006 International Conference on Image Processing: Algorithms and Systems, Neural Networks and Machine Learning Vol. 6064, February 17, 2006, SPIE-The International Society for Optics and Photonics, Bellingham, Washington, USA., pp: 1-11.

Dabov, K., A. Foi, V. Katkovnik and K. Egiazarian, 2007. Image denoising by sparse 3-D transform-domain collaborative filtering. IEEE Trans. Image Proces., 16: 2080-2095.

Donoho, D.L. and J.M. Johnstone, 1994. Ideal spatial adaptation by wavelet shrinkage. Biometrika, 81: 425-455.

Donoho, D.L., I.M. Johnstone, G. Kerkyacharian and D. Picard, 1995. Wavelet shrinkage: Asymptopia?. J. Royal Stat. Soc. Ser. B. Methodol., 57: 301-337.

Durand, F. and J. Dorsey, 2002. Fast bilateral filtering for the display of high-dynamic-range images. Proceedings of the 29th Annual Conference on Computer Graphics and Interactive Techniques, (SIGGRAPH '02), San Antonio, Tex, USA., July 2002,-pp: 257.
Eisemann, E. and F. Durand, 2004. Flash photography enhancement via. intrinsic relighting. Proceedings of the 2004 International Conference on ACM Transactions on Graphics (TOG) Vol. 23, August, 8-12, ACM, Los Angeles, California, pp: 673-678.

Elad, M. and M. Aharon, 2006a. Image denoising via. learned dictionaries and sparse representation. Proceedings of the 2006 IEEE International Conference on Computer Society Computer Vision and Pattern Recognition (CVPR'06) Vol. 1, June 17-22, 2006, IEEE, New York, USA., pp: 895-900.

Elad, M. and M. Aharon, 2006b. Image denoising via. sparse and redundant representations over learned dictionaries. IEEE Trans. Image Process., 15: 3736-3745.

Elad, M., 2002. On the origin of the bilateral filter and ways to improve it. IEEE. Trans. Image Process., 11: $1141-1151$.

Elad, M., 2005. Retinex by two bilateral filters. Proceedings of the 5th International Conference on Scale-Space Theories in Computer Vision, April 7-9, 2005, Springer, Berlin, Germany, pp: 217-229.

Fattal, R., Agrawala, M. and S. Rusinkiewicz, 2007. Multiscale shape and detail enhancement from multi-light image collections. Proceedings of the 2007 International Conference on ACM Transactions on Graphics (TOG) Vol. 26, August 5-9, 2007, ACM, New York, USA., pp: 1-9.

Hirakawa, K. and T.W. Parks, 2006. Image denoising using total least squares. IEEE. Trans. Image Process., 15: 2730-2742.

Kervrann, C. and J. Boulanger, 2006. Optimal spatial adaptation for patch-based image denoising. IEEE. Trans. Image Process., 15: 2866-2878.

Lee, J.S., 1983. Digital image smoothing and the sigma filter. Comput. Vision Graphics Image Process., 24 : 255-269.

Luisier, F., T. Blu and M. Unser, 2007. A new SURE approach to image denosing: Interscale orthonormal wavelet thresholding. IEEE Trans. Image Process., 16: 593-606.

Lyu, S. and E.P. Simoncelli, 2007. Statistical Modeling of Images with Fields of Gaussian Scale Mixtures. In: Advances in Neural Information Processing Systems 19, Scholkopf, B., J. Platt and T. Hoffman (Eds.). MIT Press, Cambridge, Massachusetts, USA., ISBN:13-978-0-262-19568-3, pp: 945-952. 
Mairal, J., M. Elad and G. Sapiro, 2008. Sparse representation for color image restoration. IEEE Trans. Image Process., 17: 53-69.

Paris, S. and F. Durand, 2006. A fast approximation of the bilateral filter using a signal processing approach. Proceedings of the 9th European Conference on Computer Vision, May 7-13, 2006, Springer, Berlin, Germany, pp: 568-580.

Pizurica, A. and W. Philips, 2006. Estimating the probability of the presence of a signal of interest in multiresolution single-and multiband image denoising. IEEE. Trans. Image Process., 15: 654-665.

Portilla, J., V. Strela, M.J. Wainwright and E.P. Simoncelli, 2003. Image denoising using scale mixtures of Gaussians in the wavelet domain. IEEE Trans. Image Process., 12: 1338-1351.

Sendur, L. and I.W. Selesnick, 2002b. Bivariate shrinkage functions for wavelet-based denoising exploiting interscale dependency. IEEE Trans. Signal Proc., 50: 2744-2756.

Sendur, L. and I.W. Selesnick, 2002a. Bivariate shrinkage with local variance estimation. IEEE. Signal Process. Lett., 9: 438-441.
Smith, S.M. and J.M. Brady, 1997. SUSAN-A new approach to low level image processing. Int. J. Comput. Vision, 23: 45-78.

Sochen, N., R. Kimmel and A.M. Bruckstein, 2001. Diffusions and confusions in signal and image processing. J. Math. Imaging Vision, 14: 195-209.

Spira, A., R. Kimmel and N. Sochen, 2007. A short-time Beltrami kernel for smoothing images and manifolds. IEEE. Trans. Image Process., 16: 1628-1636.

Tomasi, C. and R. Manduchi, 1998. Bilateral filtering for gray and color images. Proceedings of the 6th International Conference on Computer Vision, January 7-7, 1998, IEEE, Bombay, India, ISBN:81-7319-221-9, pp: 839-846.

Wong, W.C., A.C. Chung and S.C. Yu, 2004. Trilateral filtering for biomedical images. Proceedings of the 2004 2nd IEEE International Symposium on Biomedical Imaging: Nano to Macro (IEEE Cat No. 04EX821), April 18, 2004, IEEE, Arlington, Virginia, USA., pp: 820-823.

Yaroslavsky, L., 1985. Digital Picture Processing: An Introduction. Springer, Berlin, Germany, ISBN:978-3-642-81929-2, Pages: 276. 